\title{
SDSS J114818.18-013823.7: Forming Compact Dwarf Galaxy through the Dwarf-Dwarf Merger
}

\section{N. Chhatkuli, S. Paudel, A. K. Gautam and B. Aryal}

Journal of Nepal Physical Society

Volume 7, Issue 2, June 2021

ISSN: 2392-473X (Print), 2738-9537 (Online)

Editors:

Dr. Binod Adhikari

Dr. Bhawani Joshi

Dr. Manoj Kumar Yadav

Dr. Krishna Rai

Dr. Rajendra Prasad Adhikari

Mr. Kiran Pudasainee

JNPS, 7 (2), 49-57 (2021)

DOI: https://doi.org/10.3126/jnphyssoc.v7i2.38622

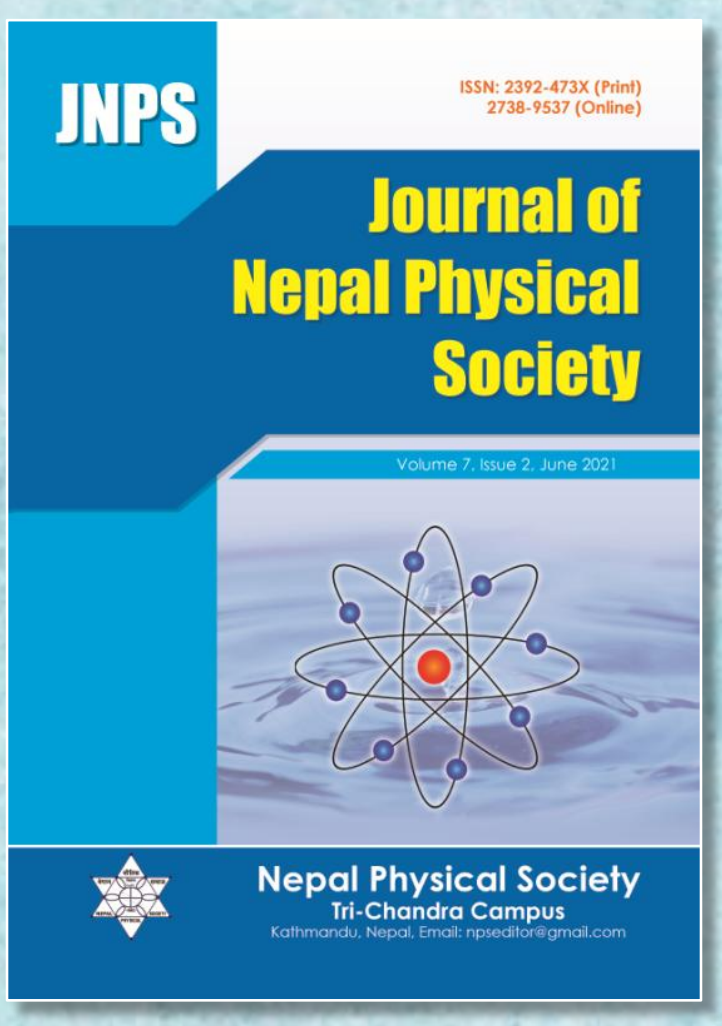

Published by:

Nepal Physical Society

P.O. Box: 2934

Tri-Chandra Campus

Kathmandu, Nepal

Email: nps.editor@gmail.com 


\title{
SDSS J114818.18-013823.7: Forming Compact Dwarf Galaxy through the Dwarf-Dwarf Merger
}

\author{
D. N. Chhatkuli ${ }^{1, ~ *}$, S. Paudel ${ }^{2}$, A. K. Gautam ${ }^{3}$ and B. Aryal ${ }^{1}$ \\ ${ }^{1}$ Central Department of Physics, Tribhuvan University, Nepal \\ ${ }^{2}$ Centre for Galaxy Evolution Research, Department of Astronomy, Yonsei University, \\ Seoul, South Korea \\ ${ }^{3}$ Bhaktapur Multiple Campus, Bhaktapur, Nepal \\ *Corresponding Email: chhatkulidn@gmail.com
}

Received: 18 April, 2021; Revised: 14 May, 2021; Accepted: 26 June, 2021

\begin{abstract}
We studied the spectroscopic properties of the low redshift $(\mathrm{z}=0.0130)$ interacting dwarf galaxy SDSS J114818.18-013823.7. It is a compact galaxy of half-light radius 521 parsec. It's r-band absolute magnitude is -16.71 mag. Using a publicly available optical spectrum from the Sloan Sky Survey data archive, we calculated star-formation rate, emission line metallicity, and dust extinction of the galaxy. Star formation rate (SFR) due to $\mathrm{H}_{\alpha}$ is found to be $0.118 \mathrm{M}_{\odot}$ year $^{-1}$ after extinction correction. The emission-line metallicity, $12+\log (\mathrm{O} / \mathrm{H})$, is 8.13 dex. Placing these values in the scaling relation of normal galaxies, we find that SDSS J114818.18-013823.7 is a significant outlier from both size-magnitude relation and SFR-B-band absolute relation. Although SDSS J114818.18-013823.7 possess enhance rate of star-formation, the current starformation activity can persist several Giga years in the future at the current place and it remains compact.
\end{abstract}

Keywords: Dwarf Galaxy, Emission line, Star Formation Rate, Metallicity, Extinction

\section{INTRODUCTION}

Dwarf galaxies are expected to be dark matter dominated objects. They are the most common galaxies in the universe. They have low surface brightness and metal poor stellar population. Their stellar masses are of the order of $10^{6}$ to $10^{9} \mathrm{M}_{\odot}$. One of the important question in understanding of formation and evolution of dwarf galaxies is how these various morphology dwarf galaxies come to exist and are they transformed from each other during the course of evolution? Dwarf galaxies are low mass objects and therefore, the capacity of binding their contents gravitationally is low as compared to their massive counterparts.

According to $\square$ CDM model of cosmology [1], the large-scale structures are formed hierarchically, and the evolution and growth of galaxies are due to merging of other galaxies [2]. Radio 21-cm observation of nearby interacting dwarf galaxies as they evolved through the merging history is yet to be explored in detail, although major mergers of galaxies are known to play an important role, particularly in the formation of the massive elliptical galaxies. In last few years, the observational evidence for mergers between dwarf galaxies has been growing. In recent years, people are working on the evolution of dwarf galaxies by dwarf-dwarf merger along with observational evidences [3, 4, 5, 6, 7, 8, 9]. Early study of dwarfdwarf interacting pairs established that the star formation rate in gas-rich isolated dwarf-dwarf pair is enhanced by a factor $\sim 2$ over isolated unpaired dwarfs having similar gas fractions and similar stellar masses alike that has been found in case of huge galaxy pairs $[10,11]$. This enhancement of SFR decreases on increasing the separation between two interacting pairs and the lack of enhancement in the SFR in unpaired dwarfs suggests that the star formation is driven by interaction. The star forming activities of the galaxies in the interstellar gas clouds depend up on various factors such as gas, dust, temperature, 
gravity, matter-radiation interaction, hydrodynamics, thermodynamics and chemical processes [12].

The galaxies with a smaller half-light radius are called compact galaxies. Many compact galaxies are found to be connected to ordinary massive galaxies [13]. It is, therefore, believed that compact galaxies are formed during the tidal interaction between a giant galaxy and a normal dwarf galaxy where the small galaxy loses its extended outer halo and survived remanent become compact dwarf galaxies. However compact dwarf galaxies are also observed in isolation, where no giant galaxies are found in the vicinity [14]. It is argued that, those isolated compact dwarfs may have formed through the merger, but this hypothesis has not been confirmed by statistically strong evidences. Therefore, a compact galaxy is selected in this work as its study gives the idea of galaxy interactions and merger. Many compact galaxies are found to be connected to ordinary galaxies, other compact galaxies, or even both of them. It is observed that the spectrum and color of the compact galaxies are wider than that of regular galaxies

Metallicity is the abundance of the heavier elements like nitrogen, oxygen, neon, silicon etc. found in a terrestrial object. Metallicity is an important parameter to know the formation and evolution of stars and galaxies. The metallicity is also related with the luminosity, decrease in luminosity of a galaxy means increase in metallicity, at fixed mass and starburst age of the galaxy [15]. In the process of merging of galaxies, the metallicity of interior zones is diluted because of the infill of metal-poor gas driven by gravity from the outskirts of the galactic disc to central region [16].

In this work, a spectroscopic study of a compact interacting dwarf SDSS J114818.18-013823.7 located in an isolated environment will be done and explore its stellar population properties to provide another unequivocal evidence of forming compact dwarf through the merger.

\section{SAMPLE SELECTION}

A low red shift $(\mathrm{z}=0.0130)$ galaxy, SDSS $\mathrm{J} 114818.18-013823.7$ is selected from the catalog of interacting dwarf galaxies [17]. It is a starburst dwarf galaxy located in the sky position R.A. $(\mathrm{J} 2000)=177.0757^{\circ}$, Dec. $(\mathrm{J} 2000)=-1.6399^{\circ}$ with a line-of-sight radial velocity $3902 \mathrm{~km} / \mathrm{s}$. It has $\mathrm{g}$ and $\mathrm{r}$ band magnitude $15.88 \mathrm{mag}$ and $15.66 \mathrm{mag}$ respectively, and total absolute B-band magnitude is $-17.21 \mathrm{mag}$. The $g-r-i$ combined tri-color image obtained from the Legacy survey is shown in Figure 1. An extended tail like structure can be seen along the major axis of the galaxy. The bluest starburst region is misplaced from the center of the galaxy. Interestingly, it can be noticed that outer extended low-surface brightness part is also blue and asymmetric morphology.

The SDSS J114818.18-013823.7 is overall a compact galaxy, the derived half-light radius is 2.1 arcsecond, that corresponds a sky-projected physical radius 530 parsec (pc). It is located in an isolated environment where we find no galaxy within a $500 \mathrm{kpc}$ sky-projected radius.

The SDSS has targeted the central region of SDSS J114818.18-013823.7 to take an optical spectrum. The SDSS fiber has an aperture of 3 arcsecond diameter, which covers a half of the SDSS J114818.18-013823.7 area.

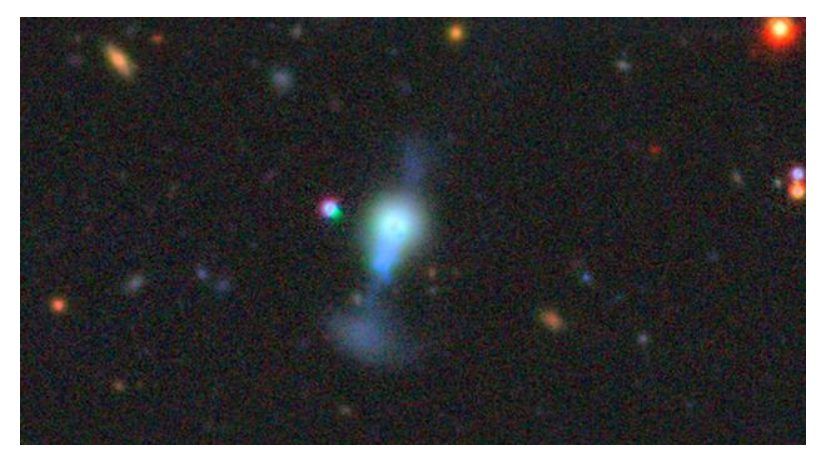

Fig. 1: Optical view of SDSS J114818.18-013823.7. The image is obtained from the SDSS sky-server.

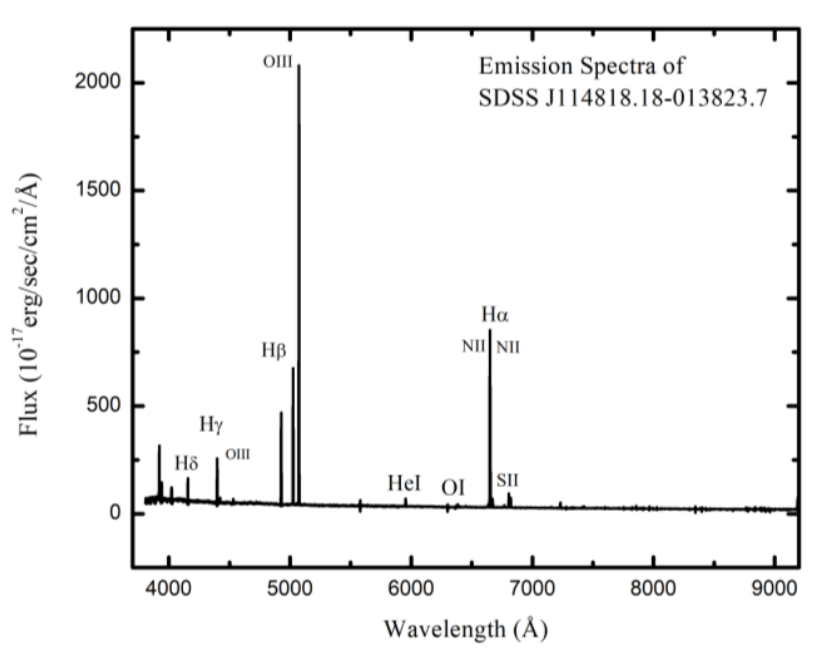

Fig. 2: Optical spectrum of SDSS J114818.18-013823.7 which is plotted by using SDSS archival data. Position of the emission lines $\mathrm{H}_{\alpha}, \mathrm{H}_{\beta}, \mathrm{H}_{\psi}, \mathrm{H}_{\delta}$, Oll doublet, NII doublet, $\mathrm{Hel}, \mathrm{OI}$ and $\mathrm{SII}$ are identified. The $\mathrm{X}$-axis is restframe wavelength and $Y$-axis is flux. 
The SDSS optical spectrum of the galaxy SDSS J114818.18-013823.7 in the wavelength range of (3757-9090) $\AA$ is shown in the Figure 2. Several characteristic lines can be seen in the spectrum and some of the prominent emission lines, $\mathrm{H}_{\alpha}, \mathrm{H}_{\beta}, \mathrm{H}_{\gamma}$, $\mathrm{H}_{\delta}$, OIII doublet, NII doublet, HeI, OI and SII are highlighted. The strongest emission line is OIII, and $\mathrm{H}_{\alpha}$ is the strongest among the Balmer lines. From this spectrum, it is clear that the galaxy is emission type galaxy.

\section{MATERIALS AND METHODS}

To measure morphological and chemical parameters of the galaxy, the SDSS archival database (www.sdss.org/collaboration/credits.html) is used. The SDSS DR12 data was extracted from the FITS file format by using software ALADIN. The galaxy spectra were obtained by plotting the data using another software ORIGIN. Then the gaussian fits were performed on the prominent emission lines by inspection. The continuum flux is subtracted by taking a median of flux value of spectrum range \pm 100 from the center of the emission line and then each line is modelled. The gaussian curves so obtained are explained by a mathematical expression called Gaussian distribution function [18] which is given by

$$
\mathrm{f}_{\mathrm{G}}(\mathrm{x})=\frac{1}{\sqrt{2 \pi \sigma^{2}}} \mathrm{e}^{\frac{-(\mathrm{x}-\mu) 2}{2 \sigma^{2}}}
$$

Where, $x$ is a normal random variable, $\mu$ is the mean and $\sigma$ is the standard deviation of the distribution. The broadening of the emission lines is due to Doppler's broadening. In this work, 10 prominent emission lines were selected and the gaussian parameters like Full Width Half Maxima (FWHM), area, height, peak intensity, wavelength corresponding to the peak intensity, regression coefficient of each of the gaussian curves were noted in a table and the corresponding responsible element for the emission will be identified. Finally, the emission line due to emission of $\mathrm{H}_{\alpha}$ will be identified and using its area, the star formation rate (SFR) of the galaxy will be calculated before and after extinction correction. In this work, the $\mathrm{H} \alpha$ luminosity is used as the main calculator of the instantaneous Star Formation Rate of the galaxy. The process of recombination of ionized hydrogen in gas clouds and the dust produces $\mathrm{H} \alpha$ line flux. The $\mathrm{O}$ and $\mathrm{B}$ type stars produce enormous radiation field that ionizes the hydrogen and several emission lines are produced due to recombination of the free electrons with the ionized hydrogen. $\mathrm{H} \alpha$ is one of the most luminous line in visible region and KennicuttSchmidt empirical relation can be used to calculate SFR using the Ho emission line flux [19].

$$
\operatorname{SFR}\left(\mathrm{M}_{\Theta} \text { year }^{-1}\right)=7.9 \times 10^{-42} \sum \mathrm{L}\left(\mathrm{H}_{\alpha}\right)\left(\operatorname{ergs~s}^{-1}\right) \ldots
$$

Where, $\sum \mathrm{L}\left(\mathrm{H}_{\alpha}\right)$ is the total luminosity of $\mathrm{H}_{\alpha}$ line which will be calculated by using area of the Gaussian fit of $\mathrm{H}_{\alpha}$ line. $\sum \mathrm{L}\left(\mathrm{H}_{\alpha}\right)=$ Area of Gaussian fit $\times 10^{-17} \times 4 \pi \mathrm{R}^{2}\left(\mathrm{ergs} \mathrm{s}^{-1}\right)$. $\mathrm{R}$ is the radius of the sphere which is calculated as $\mathrm{R}=\mathrm{D} \times 3.08 \times 10^{24}$ $\mathrm{cm}$. Here, $\mathrm{D}$ is luminosity distance in Mpc. The $\mathrm{H \alpha}$ emission line is one of the best estimators of SFR because, it depends up on very young stars only, and is independent of recent star formation history. Moreover, $\mathrm{H} \alpha$ traces directly the SFR and has very low dependence on metallicity or on ionization conditions of the gas cloud.

The emission line metallicity of the galaxy can be calculated using ratio of NII and $\mathrm{H} \alpha$ emission line flux [20], using an equation

$$
12+\log (\mathrm{O} / \mathrm{H})=8.743+0.462 \times \log \left(\mathrm{NII} / \mathrm{H}_{\alpha}\right) \ldots
$$

The difference in extinction between $\mathrm{B}$ band and $\mathrm{V}$ band is $\mathrm{E}(\mathrm{B}-\mathrm{V})=1.97 \times \log \left(\mathrm{c} / \mathrm{c}_{0}\right)$. Where, $\mathrm{c}=$ $\mathrm{H}_{\alpha} / \mathrm{H}_{\beta}$ is Blamer decrement. The theoretical value of $c_{0}$ is 2.86 [19] for an electron temperature of $10^{4} \mathrm{~K}$. Extinction coefficient is now given by

$$
\mathrm{A}(\mathrm{H} \alpha)=2.45 \mathrm{E}(\mathrm{B}-\mathrm{V}) \ldots(4)
$$

\section{RESULTS AND DISCUSSION}

\subsection{Fitted Gaussian Curves}

The Gaussian curves representing Gaussian fits of ten selected emission lines $\mathrm{HeI}, \mathrm{H}_{\delta} . \mathrm{H}_{\gamma}, \mathrm{H}_{\beta}$, $\mathrm{OIII}_{4960 \text {, }}$ $\mathrm{OIII}_{5008}, \mathrm{OI}, \mathrm{H}_{\alpha}$, NII and SII are shown in the Figures 3 and 4 below. The statistical error bars $( \pm 1 \sigma)$ are shown in each curve. From the figure, it can be seen that the observed data agrees almost perfectly with the Gaussian distribution. The Balmer lines i.e., $\mathrm{H}_{\delta}, \mathrm{H}_{\gamma}, \mathrm{H}_{\beta}$ and $\mathrm{H}_{\alpha}$ that lie in the wavelength range of (4096 to 6574) $\AA$ are shown in Figure 3. Balmer lines appear due to abundance of hydrogen in the galaxy which appear relatively stronger as compared to other elements and are often used to identify the unknown objects, to determine the radial velocity, distance, star formation rate and so on. Specially, $\mathrm{H}_{\alpha}$ is used to calculate SFR of the gas-rich galaxies. 

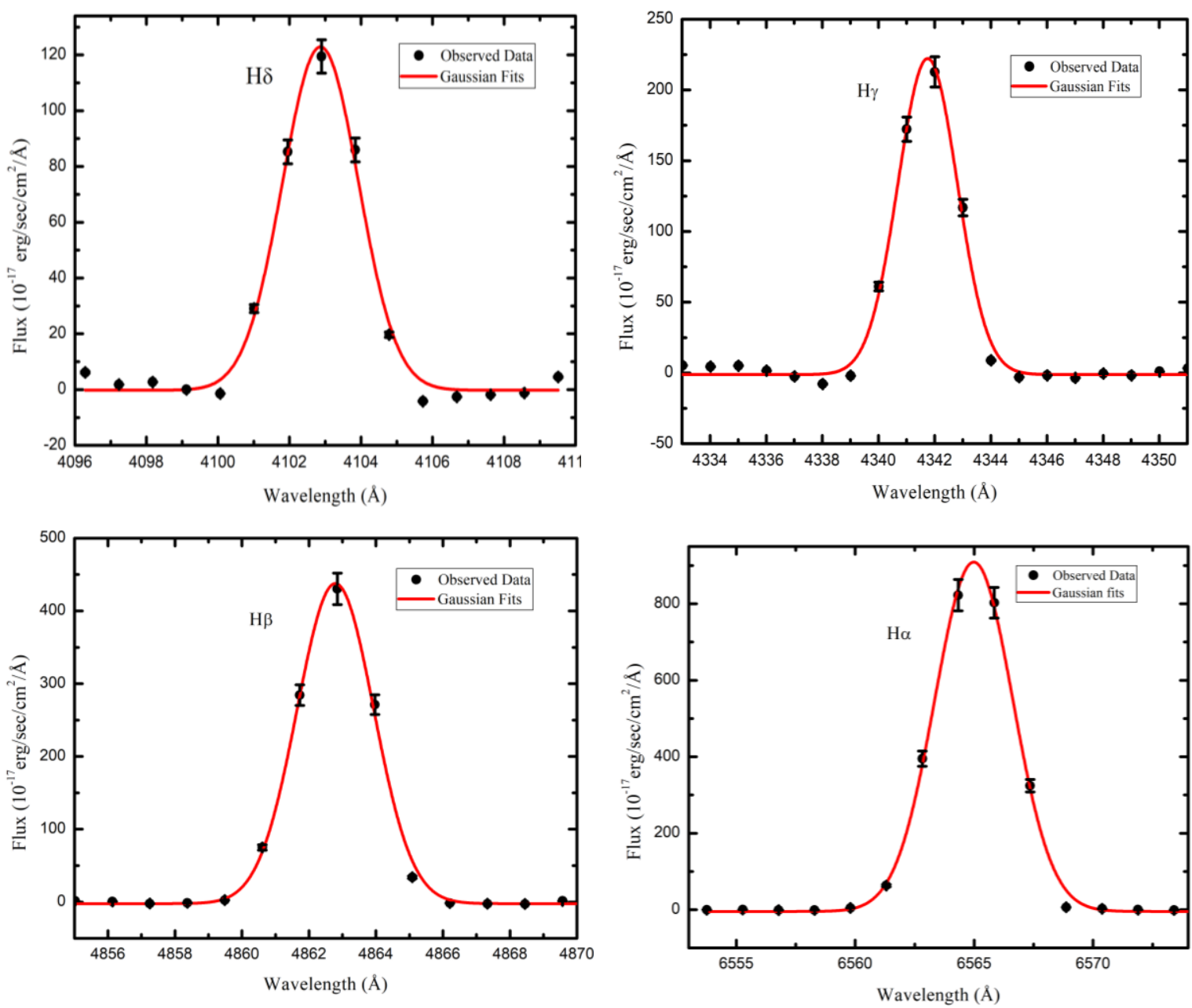

Fig. 3: Gaussian fitting procedure of the prominent emission spectral lines is shown in the figure. Four Balmer lines are: $\mathrm{H}_{\delta} . \mathrm{H}_{\gamma}, \mathrm{H}_{\beta}$, and $\mathrm{H}_{\alpha}$. Solid curves represent Gaussian fits. We show a conservative estimate of the flux error in the plot, i.e., $10 \%$ of the observed flux provided by SDSS webpage (https://www.sdss.org/dr15/spectro/caveats/). Error bars represent the $\pm 1 \sigma$ statistical errors. The major cause of broadening of characteristic line is due to the Doppler broadening. The wavelengths given in the X-axis are redshift corrected.

Figure 4 shows the emission lines due to HeI, OIII doublet, OI, NII and SII in the wavelength range (3883 to 6729) A. OIII doublet produces two lines at wavelengths $4960.1 \AA$ and $5008.3 \AA$ respectively. The emission line fluxes of heavier elements OIII and NII are used to calculate the emission line metallicity.

\subsection{Observation Table}

All the ten emission spectral lines $\mathrm{HeI}, \mathrm{H}_{\delta} . \mathrm{H}_{\gamma}, \mathrm{H}_{\beta}$, $\mathrm{OIII}_{4960}, \mathrm{OIII}_{5008}, \mathrm{OI}, \mathrm{H}_{\alpha}$. NII and SII of the galaxy SDSS J114818.18-013823.7 are enlisted in the Table 1 along with their Gaussian parameters. The first, second, third and fourth columns represent serial number, the elements responsible for the emission of lines, the wavelength $\left(\lambda_{\mathrm{p}}\right)$ corresponding to the peak intensity, the value of peak intensity $\left(\mathrm{I}_{\mathrm{p}}\right)$ in $\mathrm{erg} / \mathrm{s} / \mathrm{cm}^{2} / \AA$ respectively. Similarly, fifth, sixth, seventh eighth and nineth columns represent full width half maximum (FWHM), area, height of Gaussian curve, offset and coefficient of regression of the fitted data respectively. We can see that the line due to $\mathrm{OIII}_{5008}$ seems to be the most intense $\left(2036.4 \mathrm{erg} / \mathrm{s} / \mathrm{cm}^{2} / \AA\right)$ corresponding to the wavelength of $5008.3 \AA$. $\mathrm{H}_{\alpha}$ is the second strongest line $\left(822.5 \mathrm{erg} / \mathrm{s} / \mathrm{cm}^{2} / \AA\right)$ at $6564.3 \AA$. The weakest line is OI $\left(5.3 \mathrm{erg} / \mathrm{s} / \mathrm{cm}^{2} / \AA\right)$ at $6366.4 \AA$. The small values of FWHM (2.30 4.01) $\AA$ of the Gaussian curves indicate the characteristic peaks. The value of FWHM is maximum (4.01 $\AA$ ), for NII which indicates that the observed data is in excellent agreement with the Gaussian distribution. 

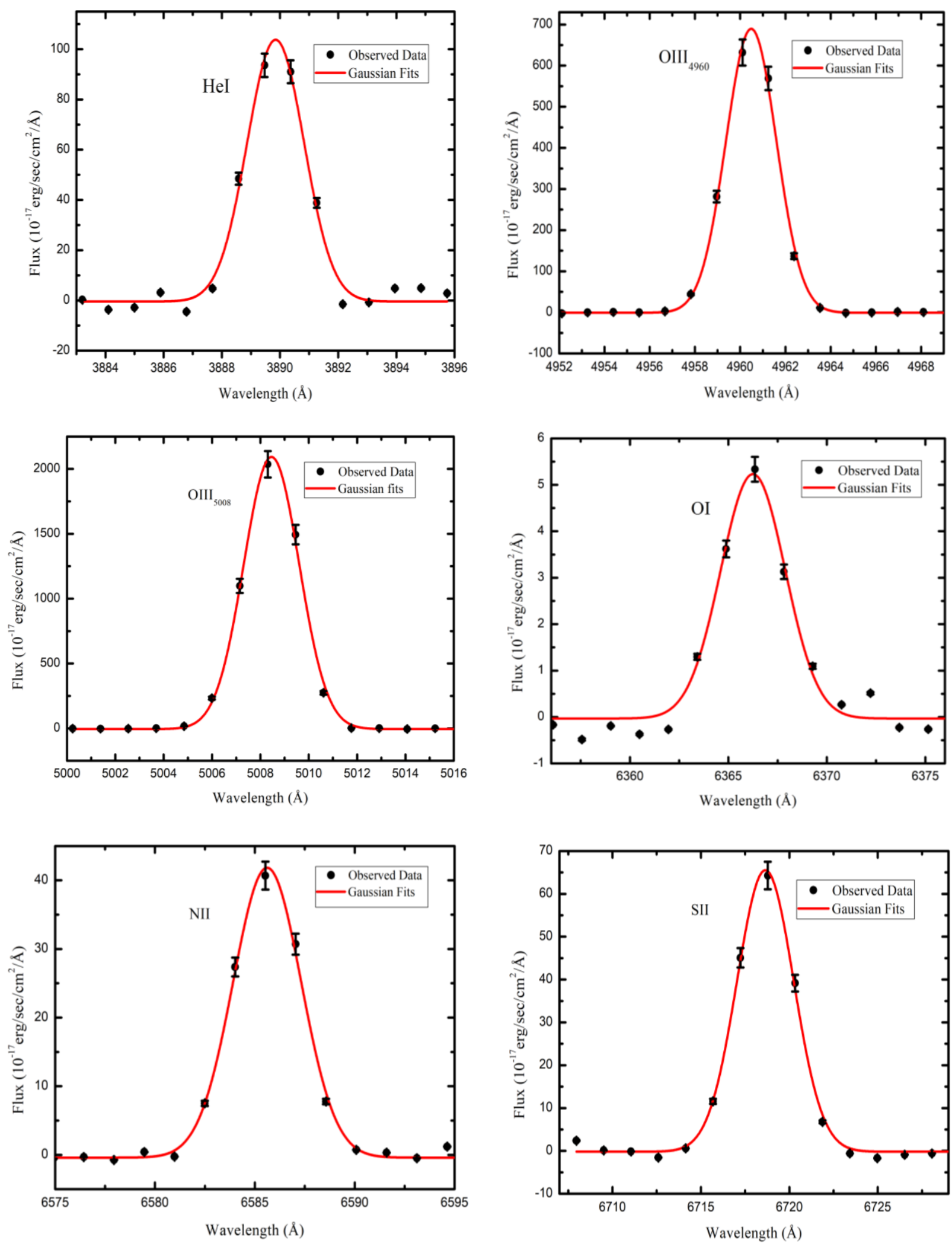

Fig. 4: Second six characteristic peaks of the elements Hel, OIII ${ }_{4960}, \mathrm{OIII}_{5008}$, OI, NII and SII of the SDSS DR12 spectra of interacting dwarf galaxy SDSS J114818.18-013823. Error bars represent the $\pm 1 \sigma$ statistical errors. The major cause of broadening of characteristic line is due to the Doppler broadening. The wavelengths given in the X-axis are redshift corrected. 
Table 1: This table enlists the Gaussian parameters of the selected characteristic emission lines of spectra of interacting dwarf galaxy SDSS J114818.18-013823.7 shown in Figure 3 and 4.

\begin{tabular}{|c|c|c|c|c|c|c|c|c|}
\hline S.N. & Element & $\lambda_{\mathrm{p}}(\AA)$ & $\mathbf{I}_{\mathrm{p}}$ & $\begin{array}{c}\text { FWHM } \\
\text { (̊) }\end{array}$ & $\underset{\left(\mathrm{erg} / \mathrm{s} / \mathrm{cm}^{2} / \AA\right)}{\text { Area }}$ & $\begin{array}{c}\text { Height } \\
\left(\mathrm{erg} / \mathrm{s} / \mathrm{cm}^{2} / \AA\right)\end{array}$ & Offset & $\mathbf{R}^{2}$ \\
\hline 1. & $\mathrm{HeI}$ & 3889.5 & 93.6 & 2.30 & 255.85 & 104.18 & -0.379 & 0.986 \\
\hline 2. & $\mathrm{H}_{\delta}$ & 4102.9 & 119.5 & 2.50 & 327.61 & 123.12 & +0.029 & 0.990 \\
\hline 3. & $\mathrm{H}_{\gamma}$ & 4342.0 & 212.8 & 2.49 & 591.04 & 223.27 & +0.256 & 0.993 \\
\hline 4. & $\mathrm{H}_{\beta}$ & 4862.8 & 430.4 & 2.70 & 1261.05 & 439.90 & +0.058 & 0.997 \\
\hline 5. & OIII & 4960.1 & 631.9 & 2.62 & 1927.93 & 690.34 & -0.384 & 0.998 \\
\hline 6. & OIII & 5008.3 & 2036.4 & 2.70 & 6028.07 & 2098.08 & -0.156 & 0.998 \\
\hline 7. & OI & 6366.4 & 5.3 & 3.87 & 21.65 & 5.26 & +0.100 & 0.949 \\
\hline 8. & $\mathrm{H}_{\alpha}$ & 6564.3 & 822.5 & 3.86 & 3756.55 & 913.60 & -0.655 & 0.998 \\
\hline 9. & NII & 6585.5 & 40.7 & 4.01 & 180.35 & 42.23 & -0.090 & 0.996 \\
\hline 10. & SII & 6718.8 & 64.3 & 3.78 & 264.10 & 65.70 & +0.136 & 0.995 \\
\hline
\end{tabular}

In the $8^{\text {th }}$ column, we can see both positive and negative values of the Gaussian offset which shows the skewness of the data. The Gaussian distributions of the selected lines of $\mathrm{H}_{\delta} . \mathrm{H}_{\gamma}, \mathrm{H}_{\beta}$, OI and SII have positive value of the offset, thereby showing that the curves have a positive skewness. This indicates that the curves are not symmetric and the right-hand tail is longer than the left-hand tail. Likewise, the negative values of Gaussian offset of HeI, OIII doublet, $\mathrm{H}_{\alpha}$ and NII indicate the negative skewness of the curves and the left-hand tail is longer than the right-hand tail. The coefficient of regression is represented by $R^{2}$ that measures how close the data are to the fitted curve. From the $9^{\text {th }}$ column of table 1 , it can be seen that the value of $\mathrm{R}^{2}$ is more than $98 \%$ except for OI suggesting that the fitted data agrees almost perfectly with Gaussian distribution.

\subsection{Calculation}

\section{Star Formation Rate}

Area of Gaussian curve for $\mathrm{H}_{\alpha}$ line $=3756.55 \times\left(10^{-}\right.$ $\left.{ }^{17} \mathrm{erg} / \mathrm{s} / \mathrm{cm}^{2} / \AA\right)$. Using the adopted distance to the galaxy $\mathrm{D}=55.44 \mathrm{Mpc}$ and $\mathrm{H}_{\alpha}$ line flux, Star Formation Rate (SFR) of the galaxy can be calculated by using equation (2). Therefore, SFR = $0.109 \mathrm{M}_{\odot}$ year $^{-1}$.

\section{Metallicity}

Line ratio $\mathrm{H}_{\alpha} / \mathrm{H}_{\beta}=2.98$ indicating that there is internal extinction. The line ratio NII/ $\mathrm{H}_{\alpha}=0.048$.
Line metallicity can be calculated by using equation 3 as $12+\log (\mathrm{O} / \mathrm{H})=8.13$ dex.

\section{SFR after Extinction Correction}

From above calculation, Blamer decrement is given by, $\mathrm{c}=\mathrm{H}_{\alpha} / \mathrm{H}_{\beta}=2.98$. Now, using equation (4), extinction coefficient is calculated to be, $\mathrm{A}(\mathrm{H} \alpha)=$ $0.089408 \mathrm{mag}$.

Again, we have A $(\mathrm{H} \alpha)=-2.5 \log$ $(\mathrm{F}(\mathrm{H} \alpha)$ obs $/ \mathrm{F}(\mathrm{H} \alpha) \mathrm{em})$. Using the value of observed flux $\mathrm{F}(\mathrm{H} \alpha) \mathrm{obs}=3756.55 \mathrm{erg} / \mathrm{s} / \mathrm{cm}^{2} / \AA$, in this equation the emitted flux is calculated to be

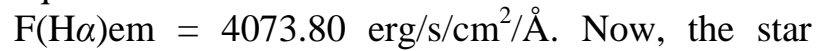
formation rate after extinction correction is calculated to be, SFR $=0.118 \mathrm{M}_{\odot}$ year $^{-1}$. It can be seen clearly that the SFR of the Galaxy before extinction is $0.108 \mathrm{M}_{\odot}$ year $^{-1}$ and that after extinction correction is $0.118 \mathrm{M}_{\odot}$ year ${ }^{-1}$. i.e.; the SFR after extinction correction is more than its previous value, which means that observed flux of the galaxy is less than the actual value of the flux. Similar work was done by Chhatkuli et al. [21] for an interacting dwarf galaxy NGC 2604, and calculated the SFR to be $0.0927 \mathrm{M}_{\odot}$ year ${ }^{-1}$, that indicates that this galaxy has higher rate of star formation. Blue compact dwarf galaxies are mostly found in isolation but they rarely go through the interaction. Recently, VCC 484 located in the Virgo cluster has been identified as merging blue compact dwarf, but detailed radio and optical imaging study 
revealed that VCC 484 no longer become compact dwarf after a few Mega year of evolution time [22]. NGC 4449 is a nearest interacting dwarf which show burst of star-formation with an ongoing starformation rate of $0.5 \mathrm{M}_{\odot}$ year $^{-1}$ [23]

\subsection{Scaling Relation}

Galaxy size-mass relation has been a standard tool to study galaxy formation and evolution mechanism [24]. In Figure 5, two scaling relations between size and r-band magnitude, and SFR and B-band magnitude are shown. It is clear from the left panel plot that SDSS J114818.18-013823.7 is a compact galaxy, where it is located significantly outside of the main locus of size-magnitude relation defined

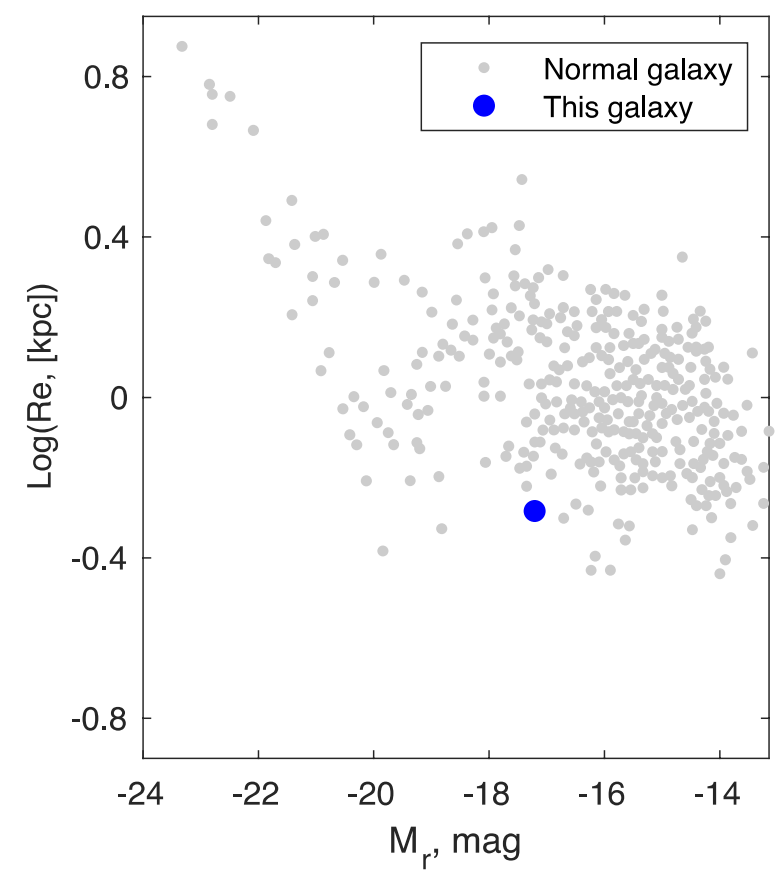

by normal galaxy's points. The right panel plot shows a relation between B-band absolute magnitude and $\log$ (SFR) of star-forming galaxies, where our galaxy the SDSS J114818.18-013823.7 is identified with a blue symbol. The comparison sample galaxies, gray symbol data point, are local volume star-forming galaxies [25]. As compared to the normal star-forming galaxies, SDSS J114818.18-013823.7 has enhanced star-formation activity. It has current SFR $0.118 \mathrm{M}_{\odot}$ year $^{-1}$. The catalog value of neutral hydrogen gas mass is $6.4 \mathrm{x}$ $10^{8} \mathrm{M}_{\odot}$. This implies that current star-formation activity can persist several Giga years in the future at the current pace.

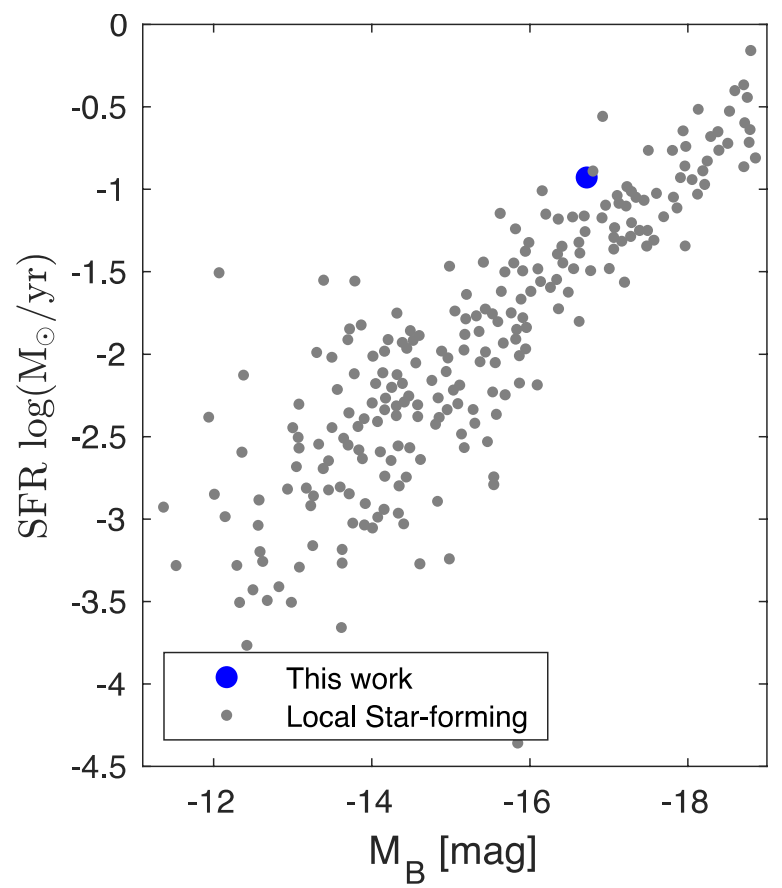

Fig. 5: Size-magnitude relation in Left and SFR-B-band magnitude relation in right. SDSS J114818.18-013823.7 is shown in blue symbol. The gray symbol represents comparison sample taken from Paudel et al., 2011 (left) and Lee et al., (2009) for the right.

\section{CONCLUSION}

In this work, we studied the spectroscopic properties of the low redshift $(\mathrm{z}=0.0130)$ interacting dwarf galaxy SDSS J114818.18013823.7 which provides another unequivocal evidence of forming compact dwarf galaxy through the merger. Ten prominent emission lines were noticed from the optical spectra of the galaxy and following conclusions are drawn.

1. The responsible elements for the observed emission lines are $\mathrm{HeI}, \mathrm{H}_{\delta} . \mathrm{H}_{\gamma}, \mathrm{H}_{\beta}, \mathrm{OIII}_{4960 \text {, }}$ $\mathrm{OIII}_{5008}, \mathrm{OI}, \mathrm{H}_{\alpha}$. NII and SII.
2. $\mathrm{OIII}_{5008}$ is the most intense $\left(2036.4 \mathrm{erg} / \mathrm{s} / \mathrm{cm}^{2} / \AA\right)$ line corresponding to the wavelength of 5008.3 $\AA, \mathrm{H}_{\alpha}$ is the second strongest line $(822.5$ $\left.\mathrm{erg} / \mathrm{s} / \mathrm{cm}^{2} / \AA\right)$ at $6564.3 \AA$ and the weakest is OI $\left(5.3 \mathrm{erg} / \mathrm{s} / \mathrm{cm}^{2} / \AA\right)$ at $6366.4 \AA$.

3. The emission lines are fitted with gaussian profile and the coefficient of regression of all the emission lines is greater than $98 \%$ except for OI, indicating that the lines are in almost perfect agreement with the Gaussian distribution. The FWHM is found to be less than $5 \AA$. The broadening of the lines is due to Doppler's broadening. 
4. Star formation rate (SFR) calculated from $\mathrm{H}_{\alpha}$ emission line is found to be $0.118 \mathrm{M}_{\odot}$ year $^{-1}$ after extinction correction.

5. The galaxy SDSS J114818.18-013823.7 is a compact galaxy having enhanced star formation activities as compared to the normal star forming galaxies and current SFR indicates that current star-formation rate can be prolonged for several Giga year in time and the galaxy remains compact due to central star-formation.

\section{ACKNOWLEDGEMENTS}

We are thankful to the anonymous referee for her/his constructive comments to improve the manuscript. D. N. Chhatkuli acknowledges the University Grants Commission of Nepal, for financial support by providing $\mathrm{PhD}$ Fellowship and Research Support Grant (Award No.: PhD-75/76-S \& T-13) to carry out this research. This study is based on the archival images and spectra from the Sloan Digital Sky Survey (http://www:sdss.org/collaboration/ credits.html).

\section{REFERENCES}

[1] Spergel, D. N.; Bean, R.; Doré, O.; Nolta, M. R.; Bennett, C. L.; Dunkley, J.; ... \& Wright, E. L.. Three-year Wilkinson Microwave Anisotropy Probe (WMAP) observations: implications for cosmology. The Astrophysical Journal Supplement Series, 170(2): 377 (2007).

[2] Conselice, C. J.; Yang, C.; \& Bluck, A. F. The structures of distant galaxies-III. The merger history of over 20000 massive galaxies at $\mathrm{z}<$ 1.2. Monthly Notices of the Royal Astronomical Society, 394(4): 1956-1972 (2009).

[3] Amorisco, N. C.; Evans, N. W.; \& Van De Ven, $\mathrm{G}$. The remnant of a merger between two dwarf galaxies in Andromeda II. Nature, 507(7492): 335-337 (2014).

[4] Crnojević, D.; Sand, D. J.; Caldwell, N.; Guhathakurta, P.; McLeod, B.; Seth, A.; ... \& Toloba, E. Discovery of a close pair of faint dwarf galaxies in the halo of Centaurus A. The Astrophysical Journal Letters, 795(2): L35 (2014).

[5] Martinez-Delgado, D.; Romanowsky, A. J.; Gabany, R. J.; Annibali, F.; Arnold, J. A.; Fliri, J.; ... \& Merrifield, M. R.. Dwarfs gobbling dwarfs: a stellar tidal stream around NGC 4449 and hierarchical galaxy formation on small scales. The Astrophysical Journal letters, 748(2): L24 (2012).

[6] Johnson, M. Determining the Nature of the Extended HI Structure around LITTLE THINGS
Dwarf Galaxy NGC 1569. The Astronomical Journal, 145(6): 146 (2013).

[7] Nidever, D. L.; Ashley, T.; Slater, C. T.; Ott, J.; Johnson, M.; Bell, E. F.; ... \& Burton, W. B. Evidence for an interaction in the nearest starbursting dwarf irregular galaxy IC 10. The Astrophysical Journal Letters, 779(2): L15 (2013).

[8] Rich, R. M.; Collins, M. L.; Black, C. M.; Longstaff, F. A.; Koch, A.; Benson, A.; \& Reitzel, D. B. A tidally distorted dwarf galaxy near NGC 4449. Nature, 482(7384): 192-194 (2012).

[9] Paudel, S.; \& Sengupta, C. UGC 4703 Interacting Pair Near the Isolated Spiral Galaxy NGC 2718: A Milky Way Magellanic Cloud Analog. The Astrophysical Journal Letters, 849(2): L28 (2013).

[10] Stierwalt, S.; Besla, G.; Patton, D.; Johnson, K.; Kallivayalil, N.; Putman, M.; ... \& Ross, G. TiNy Titans: The role of dwarf-dwarf interactions in low-mass galaxy evolution. The Astrophysical Journal, 805(1): 2 (2015).

[11] Ellison, S. L.; Mendel, J. T.; Patton, D. R.; \& Scudder, J. M. Galaxy pairs in the Sloan Digital Sky Survey-VIII. The observational properties of post-merger galaxies. Monthly Notices of the Royal Astronomical Society, 435(4): 3627-3638 (2013).

[12] Krumholz, M. R.; Klein, R. I.; \& McKee, C. F. Radiation-hydrodynamic simulations of the formation of orion-like star clusters. II. The initial mass function from winds, turbulence, and radiation. The Astrophysical Journal, 754(1): 71 (2012).

[13] Labarrere, C. A.; Woods, J. R.; Hardin, J. W.; Campana, G. L.; Ortiz, M. A.; Jaeger, B. R.; ... \& Wozniak, T. C.. Early prediction of cardiac allograft vasculopathy and heart transplant failure. American Journal of Transplantation, 11(3): 528-535 (2011).

[14] Paudel, S.; Lisker, T.; Hansson, K. S. A.; \& Huxor, A. P. An isolated, compact early-type galaxy with a diffuse stellar component: Merger origin? Monthly Notices of the Royal Astronomical Society, 443(1), 446-453 (2014).

[15] Parnovsky, S. L.; Izotova, I. Y.; \& Izotov, Y. I. H $\alpha$ and UV luminosities and star formation rates in a large sample of luminous compact galaxies. Astrophysics and Space Science, 343(1): 361-376 (2013).

[16] Rupke, D. S.; Kewley, L. J.; \& Barnes, J. E. Galaxy mergers and the mass-metallicity relation: evidence for nuclear metal dilution and flattened gradients from numerical simulations. The Astrophysical Journal Letters, 710 (2): L156 (2010). 
[17] Paudel S.; Smith R.; Yoon S. J.; CalderónCastillo P.; \& Duc P. A. A Catalog of Merging Dwarf Galaxies in the Local Universe. The Astrophysical Journal Supplement Series, 237: 120 (2018).

[18] Squires G. L. \& Squires G. L. Practical physics. Cambridge University press.

https://www.cambridge.org/np/academic/subjects/ physics/general-and-classical physics/practicalphysics-4th-edition? (2001).

[19] Kennicutt Jr. and Robert C. Star Formation in Galaxies Along the Hubble Sequence. Annual Review of Astronomy and Astrophysics, 36(1): 189-231 (1998).

[20] Marino, R. A.; Rosales-Ortega, F. F.; Sánchez, S. F.; De Paz, A. G.; Vílchez, J.; Miralles-Caballero, D., ... \& Van De Ven, G. The O3N2 and N2 abundance indicators revisited: improved calibrations based on CALIFA and Te-based literature data. Astronomy \& Astrophysics, 559: A114 (2013).

[21] Chhatkuli, D.; Paudel, S.; \& Aryal, B. Study of Star Formation Rate and Metallicity of an Interacting Dwarf Galaxy NGC 2604. Journal of Institute of Science and Technology, 25(2): 55-60 (2020).

[22] Zhang, H. X.; Paudel, S.; Smith, R.; Duc, P. A.; Puzia, T. H.; Peng, E. W.; ... \& Oh, S. H. The Blue Compact Dwarf Galaxy VCC 848 Formed by Dwarf-Dwarf Merging. The Astrophysical Journal Letters, 891(1): L23 (2020).

[23] Cignoni, M.; Sacchi, E.; Aloisi, A.; Tosi, M.; Calzetti, D.; Lee, J. C.; ... \& Whitmore, B. C. Star Formation Histories of the LEGUS Dwarf Galaxies. I. Recent History of NGC 1705, NGC 4449, and Holmberg II. The Astrophysical Journal, 856(1): 62 (2018).

[24] Janz, J. \& Lisker, T. The Sizes of Early-Type Galaxies. The Astrophysical Journal, 689(1): L25-L28 (2008).

[25] Lee, J. C.; Kennicutt, R.C., Jr.; Funes, S.J.J.G.; Sakai, S.; Akiyama, S. Dwarf Galaxy Starburst Statistics in the Local Volume. The Astrophysical Journal, 692: 1305-1320 (2009). 